\title{
PSYCHOPHYSIOLOGICAL AND SUBJECTIVE RESPONSES OF A COMMUNITY SAMPLE OF VIDEO LOTTERY GAMBLERS IN GAMBLING VENUES AND LABORATORY SITUATIONS
}

Katherine M. Diskin

David C. Hodgins

Steven A. Skitch

University of Calgary

Calgary, Alberta, Canada

\begin{abstract}
Physiological and subjective arousal in lounge and laboratory conditions were explored using heart rate (HR), skin conductance (SCL) and subjective ratings for a community sample $(\mathrm{N}=30)$ of video lottery terminal gamblers (14 non-pathological gamblers [NPG] and 16 probable pathological gamblers [PPG]). For all participants, mean heart rates and ratings of subjective arousal were higher in the lounge situation, while SCL did not differ between locations. SCL increased over baseline when gambling in both situations. HR initially increased over baseline in the lounge situation only, but by the end of gambling HR increased in both situations. HR, SCL, and subjective reports were moderately correlated between venues. No correlations were found between subjective and physiological measures of arousal.
\end{abstract}


A comprehensive theory that explains the development and maintenance of problem gambling behaviour has yet to be derived. Current opinion suggests that an explanation that encompasses biological, social and psychological elements is likely to prove most useful in the understanding of problem gambling (Griffiths and Delfabbro, 2002; Sharpe, 2002). One area of interest in the development of a biopsychosocial understanding of gambling behaviour is the relationship between levels of physiological arousal and gambling. It has been generally assumed that gambling is a behaviour that results in an increase in physiological arousal, which is experienced by the gambler as excitement (Anderson and Brown, 1984). It is not clear, however, whether all forms of gambling produce similar levels of arousal. Given the variety of gambling opportunities, it is unlikely that gamblers' physiological responses are the same in all gambling situations. The game being played, the gambling venue, the size of individual wagers, and the speed of play may all affect how individuals respond both physically and cognitively to a particular gambling experience. It would be unwise to generalize about levels of physiological and perceived excitement from one type of gambling to another. It may be that people seek out particular forms of gambling that 'fit' their needs for a particular experience.

Electronic machine gambling, with its combination of continuous speed of play, attractive lights and sounds, and relatively small individual wagers, has become increasingly popular in recent years. The present study is a continuation of our examination of how people respond physiologically and subjectively to the experience of gambling on video lottery terminals (VLTs) (Diskin and Hodgins, 2003).

Several researchers have used measures of heart rate to explore physiological responses to machine gambling, with varying results. Leary and Dickerson (1985) found both regular and occasional videopoker players experienced increased heart rates when gambling in a laboratory situation, with greater increases in beats per minute (bpm) experienced by regular gamblers $(M=13.5)$ compared to occasional gamblers $(M=9.1)$. Similarly, Coloumbe, Ladouceur, Desharnais, and Jobin (1992) found increased heart rate in 12 regular $(M=12.8)$ and 12 occasional videopoker players $(M=10.9)$ when gambling in a natural environment, but the between group difference was not significant.

Among adolescent fruit machine players in a natural setting, Griffiths (1993) found heart rates increased by approximately $22 \mathrm{bpm}$ for both regular and non-regular players. Coventry and Constable (1999) found that among 32 high and low frequency fruit machine players, significant increases in heart rate during and after gambling were found only for those players who experienced a win during the session. No correlation was found between heart rate and subjective arousal. Coventry and Hudson (2001) measured heart rate for a mixed sample of 42 male and female fruit machine players. Winning was a significant predictor for heart rate increase over baseline and a significant correlation was found between subjective arousal and heart rate.

Research on blackjack gamblers has found differing increases in HR in more and less natural conditions. Anderson and Brown (1984) found large differences in heart rate 
between laboratory and casino gambling situations in a group of experienced blackjack gamblers. When participants played blackjack in the casino condition, a mean increase in heart rate of approximately $23 \mathrm{bpm}$ was found, compared to a mean increase of approximately $7 \mathrm{bpm}$ in the laboratory situation. A recent study of 10 male regular blackjack players (Meyer et al., 2000) found that when blackjack players used their own money when gambling, (the experimental condition) heart rates increased $(M=30)$ during the first 30 minutes of gambling and remained elevated through the session. No correlation between gambling severity and change in heart rate when gambling was found in the experimental (gambling) condition. In the control condition, when gamblers played for points only, a negative correlation was found between severity of gambling problems and heart rate reactivity when gambling. A recent study on expectancies in video gambling (Ladouceur, Sevigny, Blaszczynski, O’Connor and Lavoie, 2003) found that a group of occasional and regular video lottery gamblers who were told they could win up to $\$ 40.00$ when gambling in a laboratory situation experienced increased heart rate prior to and during play compared to a group told they would be playing for non redeemable credits. It was concluded that 'expectancy of winning money is a cognitive factor influencing levels of arousal’ p. 733.

Researchers exploring machine gambling have not directly compared physiological response in the laboratory with natural situations. Ladouceur, Gaboury, Bujold, Lachance and Tremblay (1991) compared laboratory and in vivo conditions for video poker players in order to explore the ecological validity of laboratory studies for video poker. When a group of regular video poker players were compared in laboratory and natural settings, they did not differ in the number of irrational vocalizations produced during gambling, the number of bets doubled, and reported motivation to gamble. However, participants did wager more money in the laboratory situation than the natural situation. The authors concluded, 'playing videopoker in the laboratory produces cognitive, behavioral and motivational phenomena which are equivalent to those observed in a natural setting' (p.115). It should be noted that their study did not include any physiological measures in either situation.

Diskin and Hodgins (2003) measured levels of skin conductance (SCL), heart rate (HR) and muscle tension (EMG) of VLT gamblers playing in a laboratory situation. A sample of 30 gamblers who met DSM-IV criteria for pathological gambling disorder, and 34 non-pathological gamblers participated in the study, in which participants played on a VLT identical to those in use in Alberta, Canada. Credits were redeemed at the end of play for a maximum cash payout of $\$ 50.00$. In the laboratory all participants experienced significant increases on all three measures of arousal during the first 2 minutes of gambling on the VLT. From baseline measurements to the first 2 minutes of gambling, mean heart rate increased $3.6(S D=4.7)$ bpm; skin conductance increased by $3.5(S D=$ 2.9) micromhos, and EMG increased $3.4(S D=6.6)$ microvolts (Diskin, 2000). There were no significant between group differences among pathological and non-pathological gamblers on the measures of physiological arousal employed, although the mean heart rates of the pathological gambling group were generally higher than the mean heart rates of then non-pathological gamblers. In addition, pathological gamblers rated their levels of subjective excitement/tension higher than non-pathological gamblers during VLT play, 
although subjective ratings were not correlated with measured physiological arousal. This research was done in a laboratory situation, in which various external stimuli associated with gambling in natural situations such as noise levels, cigarette smoke, and other gamblers were not present. Participants did not use their own money to gamble, and were limited in the amount of money they could win. It was unclear if the results, particularly the lack of between group differences, would generalize to in vivo machine gambling situations. Due to the difficulties inherent in doing research with gamblers in vivo, a small study was planned in order to develop a clearer understanding of how gamblers respond in laboratory and natural gambling situations.

The present study examined the ecological validity of laboratory research for machine gambling by exploring several areas of interest including:

1) The relationship between levels of physical arousal in gambling venues and in laboratory gambling situations for the same individuals.

2) Whether gamblers' level of gambling problems would result in differences in physiological arousal and subjective responses to gambling in natural and laboratory situations.

3) Possible relationships between reported levels of subjective arousal and measures of physiological arousal in both venues.

\section{Method}

\section{Participants}

This study was the first machine gambling research to be undertaken in a natural environment in the city of Calgary, Alberta. The number of participants in the study was limited in order to minimize disruption within the various gambling venues. Recruitment took place over approximately 8 months. In order to get a sample that would correspond as closely as possible to people who play VLTS in Calgary, the sole exclusion criterion for the study was that gamblers had to be 18 or over (the legal gambling age in Alberta). Thirty-one video lottery gamblers were recruited from three lounges in Calgary, Alberta, Canada. One participant was deleted from the analysis as he chose not to come to the University of Calgary for the laboratory component of the research. The sample consisted of 17 male and 14 female gamblers, ranging in age from 19 to 75.

Since the participants were a community sample of VLT players, there were not equivalent numbers of individuals with similar levels of gambling problems. Participants had scores on the Problem Gambling Severity Index of the Canadian Problem Gambling Index (CPGI; Ferris and Wynne, 2001) ranging from 0 to $27(M=7.3, S D=5.7)$, and scores on the South Oaks Gambling Screen (SOGS; Lesieur and Blume, 1987) ranging from 0 to $15(M=5.6, S D=2.9)$. Six participants met DSM-IV criteria for pathological gambling disorder based on the NORC DSM-IV Screen for Gambling Problems (NODS; Gerstein et al., 1999), (scores ranged from $0-10, M=2.5, S D=2.9$ ). Participants were divided into two groups based on SOGS scores. Those with a score of less than 5 on the SOGS $(n=14)$ were designated the non-pathological gambling group (NPG), even though scores of 3-4 on the SOGS may indicate potential gambling problems, and those 
who obtained a score of 5 or greater on the SOGS $(n=16)$ were designated the probable pathological gambling group (PPG). Given the sample size gamblers who scored less than 5 on the SOGS were not further differentiated. (Participants' scores on the SOGS, NODS, and CPGI are included in Appendix A).

The NPG and PPG groups did not differ significantly on gender (there were 7 females in each group) $\chi^{2}(1,29)=.117, p=.509$, or age (NPG $M=38.1, S D=16.2$, PPG $M=47.2$, $S D=13.6), t(27)=1.63, p=.115$. No significant between-group differences were found for current or past major depressive episodes, current or past alcohol abuse/dependence, current or past cannabis abuse/dependence or current or past cocaine abuse/dependence.

\section{Apparatus}

1) Procomp+ biofeedback system with heart rate/blood volume sensor and skin conductance sensors, using a Biograph software data recording and analysis program with a Toshiba laptop PC.

2) Video lottery terminals in general use in licensed premises in the city of Calgary

3) Portable CD player with earphones and a classical music CD

4) Video lottery terminal (model VLC) set in demonstration mode, located in the Addictive Behaviours Laboratory, University of Calgary. The machine was supplied by the Alberta Gaming and Lotteries Commission and is identical to those in use in the province. Credits were entered using the touch screen rather than by inserting money into the terminal.

\section{Measures}

Canadian Problem Gambling Index (CPGI; Ferris and Wynne, 2001) The Problem Gambling Severity Index of the CPGI was used. In the initial reliability and validity study Cronbach's alpha for the 9-item scale was .84 and test retest reliability (3-4 weeks) was .78.

NORC DSM-IV Screen for Gambling Problems (NODS; Gerstein et al., 1999) The NODS is an instrument with 17 items that correspond to the DSM-IV criteria for pathological gambling disorder. A small reliability and validity study revealed excellent test-retest reliability across a two to four week period (past year screen $r=.98$ ).

The South Oaks Gambling Screen (SOGS; Lesieur and Blume, 1987) is a 20- item selfreport questionnaire widely used to screen normal and clinical populations for pathological gambling. A person who receives a score of 5 or greater on the SOGS (i.e. answers 'yes' to 5 or more questions) is identified as a probable pathological gambler.

Structured Clinical Interview for the DSM-IV (SCID-I/NP; First, Spitzer, Gibbon and Williams, 1997). The Psychoactive Substance Use module, the Current Major Depressive Episode and Past Major Depressive Episode modules, and the Current Manic Episode and Past Manic Episode modules were administered. The SCID-R is a structured interview that enquires about the frequency and intensity of symptoms and provides a diagnosis. 
Perception of excitement and tension self-report checklists. These checklists were used to measure levels of subjective excitement and tension. Participants were asked to rate their perceived level of excitement, nervousness and tension at various points using a Likert type scale from 1-7(for example 1 = not at all excited, 7 = very excited).

\section{Physiological Measurements}

Heart rate was measured with a photoplethysmographic sensor using an LED photodetector with a light source and light sensor placed on the pad of the index finger of the non-dominant hand. When the body is preparing for action sympathetic nervous system activity increases (Matsumo, Walker, Walker, and Hughes, 1990) and the heart rate accelerates.

Skin conductance level was measured using self-adhesive $1 \mathrm{~cm}$ silver silver chloride electrodes placed on the thenar and hypothenar eminences of the palm. When the sympathetic nervous system is aroused sweating increases. As a result, the skin's capacity to conduct current is enhanced and SCL increases.

Physiological measurements were recorded using a Procomp+/Biograph psychophysiological data acquisition system attached to a laptop computer.

\section{Procedure}

\section{Part 1}

Participants were recruited at three local lounges that offer VLT gambling. A research assistant gathered basic demographic information from the participants, and administered the CPGI (Ferris and Wynne, 2001). Small physiological sensors measuring skin conductance and heart rate were attached to the non-dominant hand. Responses were measured for a two-minute baseline period while participants listened to classical music on headphones in order to block any sounds from the lounge. After the baseline measurement the participants were asked to choose a VLT and gamble as they normally would, using their own money, for as long as they wished (time gambled was recorded to a maximum measurement period of 20 minutes). Participants chose any game they wished to play and were free to change games during the recorded session. The time and amount of any wins greater than or equal to 50 credits during the play session were recorded. As well, the total amount of time that participants played on the VLT was noted. After they finished gambling the participants were asked to rate their feelings of excitement, nervousness, and tension when they had started and stopped gambling.

\section{Part 2}

Participants were asked to make an appointment for the second part of the study, which took place at the Addictive Behaviours Laboratory at the University of Calgary. Structured Clinical Interview modules for past and present polysubstance abuse, depression and manic episodes, the NODS, and the SOGS were administered and physiological sensors were applied to the participant's non-dominant hand. A two-minute 
baseline measurement was taken, while the participants listened to classical music on headphones. They were then instructed to play on the demonstration VLT as they normally would, including changing games if desired. Prior to beginning the gambling session the research assistant inserted credits into the demonstration machine that were equivalent to the amount the participant had used in the lounge session, and wins and total time played were noted. Levels of excitement, tension and nervousness were rated when participants finished playing. Participants were free to stop playing at any time, (maximum time allowed was 20 minutes). Any credits remaining at the end of the session were redeemed to a maximum value of $\$ 50.00$ cash. All participants who completed the study received a $\$ 50.00$ grocery gift certificate. Participants who indicated any concern about their gambling behaviour were given information on problem gambling resources in the province.

It should be noted that in both Parts 1 and 2 we were not able to video tape the session, therefore the number of 'near misses' experienced by participants could not be recorded.

\section{Results}

Before conducting further analyses, the average amount of time participants spent gambling in the laboratory and lounge venues was compared. The mean recorded gambling session in the lounge was 10.9 minutes $(S D=5.7)$; while the mean recorded gambling session in the lab was 9.9 minutes $(S D=5.1)$. No significant between location difference was found, $t(29)=0.9, p=.40$, for mean amount of time gambled for all participants. In the lounge situation there was no between group difference in the amount of time participants gambled. Non-pathological players gambled for 11.5 minutes in the lounge, while probable pathological players gambled for 10.3 minutes $(t[28]=0.6, p=$ .57). In the laboratory situation, there was a significant between group difference in the length of time participants chose to gamble. Non-pathological gamblers played for a mean of $7.3(S D=2.2)$ minutes in the laboratory situation, while probable pathological gamblers played for a mean of $11.9(S D=6.0)$ minutes in the laboratory, $t(28)=2.7, p=$. 012.

In the lounge situation, a total of 7 wins of at least 100 credits (one credit $=\$ 0.25$ ) occurred over the course of the study, while in the laboratory situation a total of 8 wins of at least 100 credits were recorded. A total of 38 wins of 50 credits or greater were recorded in the lounge venues, while 25 wins of 50 or greater credits were recorded in the laboratory. This difference was non-significant, $t(29)=1.5, p=.15$. No significant difference was found between the number of wins experienced by the PPG and NPG groups in either venue $(t[28]=1.2, p=.24$ in the lounge, $t[28]=0.31, p=.76$ in the laboratory).

Questions 1 and 2-Is there a difference in physiological and subjective arousal in laboratory and lounge situations? Are there differences in physiological and subjective arousal related to level of gambling problems?

Physiological Responses 
In order to test for possible differences in physiological responses (heart rate and skin conductance) depending on location (laboratory and lounge), task (baseline vs. gambling) and degree of gambling problem (NPG and PPG), 2 × 2 × 2 analyses of variance were employed using the between subjects factor of Gambling Group and the within subject factors of Task (baseline, first or last 30 seconds of gambling) and Location (lounge and laboratory).

\section{Heart Rate}

We examined the effect of these factors on heart rate using two analyses of variance. In the first analysis, the two levels of the Task factor were the last 30 seconds of baseline and the first 30 seconds of gambling. The analysis of variance did not produce a significant effect for Group (NPG, PPG), $F(1,28)=1.87, p=.183$, or Task (baseline, first 30 seconds gambling) $F(2,28)=0.87, p=.358$. A significant main effect for Location (lounge, laboratory), $F(1,28)=17.49, p<.001$, and a significant Task by Location interaction, $F(2,28)=8.18, p=.008$ were found. The Task by Location by Group interaction was not found to be significant $(F[1,28]=.38, p=.545)$. Examination of the main effect of Location indicated that mean heart rates were significantly higher in the lounge situation $(M=84.1, S D=2.2)$, compared to the laboratory situation $(M=77.5$, $S D=1.8)$. Examination of the Task by Location interaction indicated that mean heart rate increased from baseline to gambling in the lounge situation, but did not increase in the lab situation. Mean heart rate increased from $M=83.2(S D=12.4)$ beats per minute at baseline to $M=85.4(S D=12.6)$ for the first 30 seconds of gambling in the lounge, $t$ $(29)=2.02, p=.05$. Mean heart rate in the laboratory situation did not differ from baseline $M=78.0(S D=10.1)$ to the first 30 seconds of gambling $M=77.4(S D=10.7), t$ $(29)=.79, p=.437$, indicating that for the first 30 seconds of VLT gambling, gambling related arousal as measured by increased heart rate was found only in the lounge situation for both groups of participants.

In the second analysis, the two levels of the Task factor employed were the last 30 seconds of the baseline and the last 30 seconds of VLT gambling. Since participants were able to stop gambling at a time of their own choosing, the last 30 seconds of gambling could occur after any period of time up to 20 minutes. No significant effect was found for Group (NPG, PPG) membership $F(1,28)=1.86, p=.183$. A significant main effect of Task (baseline heart rate $M=80.4, S D=1.9$, end of gambling heart rate $M=82.1, S D=$ 1.7), $F(1,28)=5.67, p=.024$, and a significant main effect of Location (lounge $M=$ 83.7, $S D=2.1$, laboratory $M=78.9, S D=1.8), F(1,28)=9.99, p=.004$, were found. Mean heart rate was higher in the lounge situation, and heart rate increases from baseline to the end of gambling were found in both locations. The Task by Location interaction found for the analysis of the first 30 seconds of gambling was not found at the end of the gambling session, $(\mathrm{F}[1,28]=.342, \mathrm{p}=.563)$ suggesting that the participants' heart rates responded to gambling more immediately in the lounge situation, but responded in a similar fashion over a longer period of time in the laboratory. No significant Task by Location by Group interaction was found $F(1,28)=.030, p=.863$. 


\section{Skin conductance}

We examined the effects of the within subject factors of Task and Location and the between subject factor of Gambling Group on skin conductance. In the first analysis the two levels of the Task factor were the last 30 seconds of the first baseline and the first 30 seconds of VLT gambling. The $2 \times 2 \times 2$ analysis of variance for skin conductance did not produce a significant main effect for Group (NPG, PPG), $F(1,28)=1.79, p=.192$, or Location (lounge, laboratory) $F(1,28)=.06, p=.814$. A significant main effect was found for Task, $F(1,28)=15.46, p=.001$. Skin conductance increased from baseline to gambling for all participants at both locations. Mean SCL in the laboratory situation increased from $M 3.5$ (SD = 2.7) micro-mhos at baseline to $M 5.60(S D=3.7)$ when gambling. Mean SCL in the lounge situation increased from $M 3.6(S D=3.5)$ micromhos to $M 5.3(S D=5.9)$ when gambling. An increase in gambling related arousal as measured by skin conductance was found for all participants in both locations. No significant Task by Location interaction $(F[1,28]=.42, p=.525)$, or Task by Location by Group interaction $F(1,28)=.38, p=.545$ was found.

In the second analysis, the two levels of the Task factor were the last 30 seconds of the baseline and the last 30 seconds of gambling for each individual. A significant main effect for Task $F(1,28)=19.58, p<.001$ was found. As well, a significant interaction between Task, Location and Group was found $F(1,28)=6.43, p=.017$. Both groups experienced increased SCL from baseline to end of gambling, however in the laboratory situation non pathological gamblers experienced a greater increase in SCL compared to probable pathological gamblers.

We further explored the relationship between physiological measures in the two venues by conducting Pearson correlations for each measure.

Heart rates in the gambling venue and the laboratory were moderately correlated during baseline measurements, during the first 30 seconds, the first 3 minutes of gambling, and for the final 30 seconds of gambling (see Table 1). Since participants determined the amount of time they spent gambling, the number of participants in the correlation analyses decreased over time from 29 at 3 minutes, 30 seconds to 12 at 6 minutes, 30 seconds. As a result, correlation analyses after the 3-minute period involved smaller numbers of participants, and were not significant.

Skin conductance in the two venues was also moderately correlated during baseline measurements, during the first 30 seconds and at 3 and 6 minutes of gambling, and for the final 30 seconds of gambling recorded for each individual (sees Table 1).

These correlations for both physiological measures indicate a moderately strong relationship between baseline levels of arousal and gambling related reactivity in both settings.

\section{Subjective measures}

Insert Table 1 about here 
Participants were asked to rate their feelings of excitement, tension and nervousness when they started and finished gambling in both locations. The three ratings were combined into Subjective Arousal index scale scores for starting and stopping gambling in both locations. Although the subjective arousal scores were composed of only three items, ratings of reliability using Cronbach's alpha were generally good. Alpha for starting gambling in the lounge was .85, and for stopping gambling in the lounge alpha was .79. In the laboratory, the reliability for the subjective arousal index for starting gambling was alpha .89; while for stopping gambling the alpha score was .54.

A $2 \times 2 \times 2$ analysis of variance was used to examine the relationship between the between subjects factor of Gambling Group and the within subject factors of Time (starting gambling, stopping gambling) and Location (lounge and laboratory) and subjective ratings of arousal.

A 2(Group [NPG/PPG]) by 2 (Time [start/stop gambling]) by 2 (Location [lounge/lab]) analysis of variance was performed using the arousal index as a measure of gambling related subjective arousal. Significant main effects were found for Time (start gambling $M=8.9, S D=.67$, stop gambling, $M=7.0 S D=.52), F(1,28)=11.28, p=.002$, and Location (lounge $M=8.7, S D=.66$, laboratory $M=7.3, S D=.51$ ) $F(1,28)=8.72, p=$ .006. No significant effect was found for Group (PPG, NPG) $F(1,28)=.20, p=.655$. No significant Time by Location $(F[1,28]=.44, p=.518$, or Time by Location by Group $(F$ $[1,28]=2.3, p=.141$, interactions were found. Non-pathological and probable pathological gamblers did not rate their levels of subjective arousal when starting and stopping gambling differently in either situation. Subjective arousal ratings were higher in the lounge and higher when participants started to gamble. Participants reported feeling more excited, nervous and tense when they started to gamble and less aroused when they stopped.

A correlation analysis was performed for the combined subjective arousal index in the lounge and laboratory venues. Ratings of arousal in the two venues when starting gambling and when stopping gambling were moderately correlated $r(30)=.59, p=.001$, and $r(30)=.48, p=.007$ respectively. These correlations suggest that there is a moderately strong relationship between how participants rated their subjective feelings of arousal in the two locations.

Question 3 - exploration of relationships between physiological and subjective measures of arousal in both venues

A correlation analysis was performed to examine whether the combined subjective arousal index scores were correlated with physiological measures of arousal in the same situation. No significant correlations were found between subjective ratings of arousal when starting and stopping gambling and measures of heart rate and skin conductance for the first and last 30-second periods of gambling in either the lounge or laboratory situations. In the lounge situation the correlation between subjective ratings when starting gambling and heart rate for the first 30 seconds of gambling was $r(30)=.22, p=.254$, 
and the correlation for skin conductance for the same period was $r(30)=.10, p=.590$ while correlations for the last 30 seconds of gambling in the lounge and the subjective measures were $r(30)=.26, p=.154$, and $r(30)=.14, p=.450$ for heart rate and skin conductance respectively. In the laboratory situation, the correlations between subjective rating of arousal and heart rate and skin conductance when starting gambling were $r(30)$ $=.11, p=.556$, and $r(30)=.01, p=.957$ respectively, and $r(30)=.21, \mathrm{p}=.269$, and $r$ (12) $p=.541$ when stopping gambling.

\section{Other Variables}

An examination for possible relationships between the variables of age, gender, number of wins experienced in the laboratory and lounge sessions, and number of cigarettes smoked daily on subjective and physiological responses was conducted. We examined correlations between these variables and change scores (baseline to last 30 seconds of gambling) for skin conductance and heart rate in both locations, and change in ratings of subjective arousal from starting to stopping gambling in both locations. No significant correlations were found (see Table 2).

\section{Discussion}

Insert Table 2 about here

The main objective of the study was to compare measures of arousal during machine gambling in natural and laboratory situations. The two physiological measures we used, heart rate and skin conductance, performed differently in the two situations. Mean heart rates were significantly higher for all participants in the lounge settings, both at baseline and when gambling. (As noted in earlier studies, mean heart rates for the PPG group were non significantly higher than the NPG group in both situations [Sharpe, Tarrier, Schotte and Spence, 1995; Diskin and Hodgins, 2003]). For the initial 30 seconds of gambling mean heart rates increased from baseline to start of gambling for all participants in the lounge situations. An initial increase in heart rate from baseline to start of gambling in the laboratory situation was not found, although the previous study found an increase (Diskin and Hodgins 2003). When the entire period of gambling was considered, mean heart rates increased from baseline to the end of gambling in both venues for both groups of participants.

Unlike mean heart rate, skin conductance increased for all participants from baseline to the first 30 seconds of gambling in both situations. In the laboratory situation, for the last 30 seconds of gambling, skin conductance increases were greater for the non-pathological gamblers than for the problem gamblers. Additionally, probable pathological gamblers played for longer than non-pathological gamblers in the laboratory situation. This difference in length of time played may explain the difference between SCL for the two groups. If the NPG group had played as long as the PPG group a reduction in mean skin conductance might have occurred as habituation increased.

Subjective ratings of arousal were higher in the lounge situations than in the laboratory and were higher when gamblers began gambling and lower when they stopped in both 
venues. Measures of heart rate and skin conductance were not correlated with subjective ratings of arousal. This supports a similar finding in our previous study, in which subjective reports of arousal during gambling and gambling related tasks were not correlated with measures of physiological arousal for the same tasks.

When considering the ecological validity of laboratory analogue situations for machine gambling, results are mixed. For the heart rate variable, initial gambling-related increases in mean heart rate took place only in the lounge situations; however by the time participants chose to stop playing, heart rate increases did not differ significantly between venues. It should also be noted that mean heart rates were consistently higher in the lounge situations both during baseline measures and while gambling. The physical movements involved in playing the VLT were similar in both venues, so movement is an unlikely explanation for this difference. Perhaps heart rate is more responsive to situational stimuli, since for skin conductance, initial gambling related increases were similar in both venues, and baseline skin conductance did not differ between venues. An alternative explanation, supported by the work of Ladouceur et al (2003) may be related to the effect of expectancy on heart rate. In the lounge venue participants could hope to win up to $\$ 1,000.00$, while in the laboratory situation their maximum win would be $\$ 50.00$. It may be that the expectation of greater financial gain caused a greater initial increase in heart rate. The higher mean values of skin conductance for the NPG group at the end of gambling in the lab may be related to the shorter time the NPG group gambled. Perhaps skin conductance would have been more similar to that of the PPG group if they had gambled for a similar length of time. Alternatively, the lower SCL of the PPG group may be related to increased tolerance over the longer period of play.

Moderately strong correlations between the laboratory and lounge situations for all measures were obtained, a finding that is helpful in supporting the validity of laboratory research. There were moderately strong correlations between venues for heart rate, skin conductance, and subjective ratings of arousal.

As in the previous study (Diskin and Hodgins, 2003) no differences were found in physiological responses to gambling between the PPG and NPG groups, and no correlations between subjective and physiological measures were found. In the present study no between group differences were found in the reports of subjective arousal. In the earlier study pathological gamblers reporting greater feelings of subjective arousal than non-pathological gamblers during gambling and gambling related tasks. We hypothesized that the groups in the prior study might have differed in their perceptions of gambling situations. There may have been a clearer differentiation between gambling groups in the prior study, since gamblers who were classified as 'pathological' met DSMIV criteria for pathological gambling disorder. In this study, since any interested gamblers were recruited, the between group differences were not as pronounced. As discussed above, the cut off score of 5 on the SOGS was used to separate participants into PPG and NPG groups. Stinchfield (2002) has suggested that 'one can be fairly confident that the respondent is not a pathological gambler if their SOGS score is between 0 and 4: and one can be fairly confident that the respondent is a pathological gambler when their SOGS score is between 7 and 20',p. 9. Given the small size of our sample, we did not 
feel we could remove the three participants who scored 5 and 6 on the SOGS, but chose instead to designate the group who had scores of 5 or more on the SOGS as 'probable pathological gamblers'.

\section{Limitations of the study}

Initially, a great deal of difficulty was experienced in getting permission to recruit participants and carry out research in lounges. As a result, a small sample was proposed to facilitate negotiations with lounge managements. As well, we sought to minimize the amount of time spent in the lounges as well as any disruption in these venues, therefore we did not video gambling sessions and asked participants for a minimal amount of information at the gambling sites. We hope that the successful completion of this study will be helpful when approaching local gambling venues in the future. While many lounge customers expressed some interest in the study they were not willing to participate; it may be that although the participants were recruited from the community the sample may not be representative.

A significant issue when doing research with small samples is the lack of sufficient power to detect an effect if one exists. No significant between group differences on physiological measures were detected. The measures had a great deal of variability (see standard deviations). Future studies will require greater sample sizes in order to have sufficient power to detect differences should they exist. It should be noted, however, that these results are similar to those we obtained with a larger sample in the earlier study (Diskin and Hodgins, 2003).

A potential confound in this study was the effect of order of location. Participants were recruited from the gambling venues; therefore all participants gambled in the lounge first, and then participated in the second part of the study, in the laboratory. It would be preferable to be able to alternate locations in order to eliminate any effects of order on physiological or subjective responses. As well, we were not able to control for alcohol or cigarette consumption in the lounge venues, while participants did not smoke or drink alcohol in the laboratory.

We have not been able to find a solution to the issues around the use of participant's own money when gambling. While in the lounge situations participants gambled with their own money, in the laboratory participants were provided with sufficient credits to fund their gambling experience. This confound is difficult to overcome, since we were not able to give participants money to gamble with in the laboratory. As a result, they may have felt they had nothing to lose in the laboratory situation. It may be that the use of entered credits rather than cash in the laboratory may explain the longer persistence of the PPG group, since the money did not come from their own pockets. As well, due to financial considerations, the maximum payout we could offer in the laboratory was $\$ 50.00$, while in the lounge situations participants could hope for larger payouts. We did not discuss the perceived value of this potential win with the players. It may be that $\$ 50.00$ was not perceived as a significant win by some of the players. As noted, the probable pathological group played longer in the laboratory situation. Possible explanations may be that they 
hoped to win the maximum $\$ 50.00$ payout and persisted longer because the money was more important to them. Alternatively, they may have considered the potential reward insignificant and may have chosen to play till they ran out of credits.

\section{Conclusions}

All participants rated their feelings of arousal higher in the lounge situations, and reported feeling more aroused when they began a gambling session and less aroused when they finished playing. Moderately strong correlations for heart rate, skin conductance, and subjective ratings of arousal were found in laboratory and lounge situations, however, as in the previous study, no correlations were found between subjective ratings of arousal and physiological measures of arousal. Mean heart rates were higher in lounge situations, while skin conductance did not differ between locations. Heart rate was slower to increase from baseline when gambling in the laboratory, but increases were similar in the two situations by the time participants chose to stop gambling. In general, there were very minor differences between the PPG and NPG groups. The groups responded similarly both physiologically and in terms of their subjective responses to gambling. The exception to this similarity of response was the finding of higher mean SCL levels for the NPG group when stopping gambling in the laboratory.

Considering the complexities involved in performing in vivo gambling research it is likely that laboratory research will continue to be undertaken. In light of these findings, it appears that there is some justification for conducting laboratory research. Researchers who choose to use laboratory situations to study gambling will need to compensate for problems with ecological validity through the use of large, well-differentiated samples. 


\section{References}

American Psychiatric Association(APA) 1994. Diagnostic and statistical manual of mental disorders $\left(4^{\text {th }}\right.$ edn.).APA, Washington, DC.

Anderson, G. and Brown, R., 1984. 'Real and laboratory gambling, sensation seeking and arousal', British Journal of Psychology, 75, pp.401-10.

Coulombe, A., Ladouceur, R., Desharnais, R., and Jobin, J., 1992. 'Erroneous perceptions and arousal among regular and occasional video poker players', Journal of Gambling Studies, 8, pp.235-44.

Coventry, K.R., and Constable, B., 1999. 'Physiological arousal and sensation seeking in female fruit machine gamblers', Addiction, 94, pp.425-30.

Coventry, K.R., and Hudson, J., 2000. 'Gender differences, physiological arousal and the role of winning in fruit machine gamblers', Addiction, 96, pp.871-9.

Dickerson, M. 1993. 'Internal and external determinants of persistent gambling: Problems in generalizing from one form of gambling to another', Journal of Gambling Studies, 9, pp.225-45.

Diskin, K., 2000. Psychophysiological arousal in VLT gamblers, unpublished master's thesis, University of Calgary, Calgary, Alberta, Canada.

Diskin, K., and Hodgins, D., 2003. 'Psychophysiological and subjective arousal during gambling in pathological and non-pathological video lottery gamblers', International Gambling Studies, 3(1), pp.37-51.

Ferris, J., and Wynne, H., 2001. The Canadian Problem Gambling Index: Final Report, Canadian Centre on Substance Abuse.

First, M.B., Spitzer, R.L., Gibbon, M., and Williams, J.B.W., Structured Clinical Interview for DSM-IV Axis I Disorders, Research Version, Non-patient edition, (SCID-I/ NP) New York: Biometrics Research, New York State Psychiatric Institute, 1997.

Gerstein, D., Hoffman, J., Larison, C., Engelman, R. Murphy, S., Palmer, A., Chuchro, L., et al., 1999. Gambling impact and behavior study. Report to the National Gambling Impact Study Commission, April 1, 1999. Retrieved 12 April 2003 [http:www.norc.uchicago.edu/new.pdf/gamble.pdf].

Griffiths, M., 1993. 'Tolerance in gambling: An objective measure using the psychophysiological analysis of male fruit machine gamblers', Addictive Behaviours, 18, pp. 365-72.

Griffiths, M., and Delfabbro, P., 2002. 'The biopsychosocial approach to gambling: Contextual factors in research and clinical interventions', The Electronic Journal of Gambling Issues, 5. Retrieved 16 January 2002[http://www.camh.net/egambling/issue 5/index.html].

Ladouceur, R., Gaboury, A., Bujold, A., Lachance, N., and Tremblay, S., 1991. 'Ecological validity of laboratory studies of video poker gaming', Journal of Gambling Studies, 7, pp.109-17.

Ladouceur, R., Sevigny, S., Blaszczynski, A., O’Connor, K., and Lavoie, M., 2003. 'Video lottery: Winning expectancies and arousal', Addiction, 98, pp.733-8.

Leary, K., and Dickerson, M., 1985. 'Levels of arousal in high and low frequency gamblers', Behaviour Research and Therapy, 23, pp.635-40. 
Lesieur, H., and Blume, S., 1987. 'The South Oaks Gambling Screen (SOGS): A new instrument for the identification of pathological gamblers', American Journal of Psychiatry, 144, pp.1184-8.

Meyer, G., Hauffa, B., Schedlowski, M., Pawlak, C., Stadler, M., and Exton, M., 2000. 'Casino gambling increases heart rate and salivary cortisol in regular gamblers', Biological Psychiatry, 48(9), pp.948-53.

Sharpe, L., 2002. 'A reformulated cognitive-behavioural model of problem gambling A biopsychosocial perspective', Clinical Psychology Review, 22, pp.1-25.

Sharpe, L., Tarrier, N., Schotte, D., and Spence, S., 1995. 'The role of autonomic arousal in problem gambling', Addiction, 90, pp.1529-40.

Stinchfield, R., 2002. 'Reliability, validity and classification accuracy of the South Oaks Gambling Screen (SOGS)', Addictive Behaviors, 27, pp.1-19. 


\section{Table 1}

Correlations between lounge and laboratory venues for HR and SCL

\begin{tabular}{|c|c|c|c|c|c|c|}
\hline & $\begin{array}{c}\text { Baseline } \\
(2 \mathrm{~m})\end{array}$ & $\begin{array}{c}\text { First 30s } \\
\text { gambling }\end{array}$ & $\begin{array}{c}3-3.5 \mathrm{~m} \\
\text { gambling }\end{array}$ & $\begin{array}{c}6-6.5 \mathrm{~m} \\
\text { gambling }\end{array}$ & $\begin{array}{c}9-9.5 \mathrm{~m} \\
\text { gambling }\end{array}$ & $\begin{array}{c}\text { Last 30s } \\
\text { for each } \\
\text { individual }\end{array}$ \\
\hline $\begin{array}{c}\text { SCL r } \\
\mathrm{n}\end{array}$ & $\begin{array}{c}.594^{* *} \\
30\end{array}$ & $\begin{array}{c}.694^{* *} \\
30\end{array}$ & $\begin{array}{c}.682^{* *} \\
29\end{array}$ & $\begin{array}{c}.746^{* *} \\
12\end{array}$ & $\begin{array}{c}.708 \\
6\end{array}$ & $\begin{array}{c}.571^{* *} \\
30\end{array}$ \\
\hline HR r & $.682^{* *}$ & $.720^{* *}$ & $.657^{* *}$ & 417 & -.670 & $.663^{* *}$ \\
$\mathrm{n}$ & 30 & 30 & 29 & 12 & 6 & 30 \\
\hline
\end{tabular}

Note: ${ }^{* *} p<.005, * p<.05$ 
Table 2

Correlations between potential covariates and change from starting to stopping gambling

\begin{tabular}{|c|c|c|c|c|c|c|}
\hline Covariate & $\begin{array}{c}\text { HR } \\
\text { Change } \\
\text { Lounge }\end{array}$ & $\begin{array}{c}\text { SCL } \\
\text { Change } \\
\text { Lounge }\end{array}$ & $\begin{array}{c}\text { Arousal } \\
\text { Change } \\
\text { Lounge }\end{array}$ & $\begin{array}{c}\text { HR } \\
\text { Change } \\
\text { Lab }\end{array}$ & $\begin{array}{c}\text { SCL } \\
\text { Change } \\
\text { Lab }\end{array}$ & $\begin{array}{c}\text { Arousal } \\
\text { Change } \\
\text { Lab }\end{array}$ \\
\hline Age & -.335 & -.016 & -.197 & -.188 & -.197 & .144 \\
\hline Gender & -.186 & -.140 & .056 & -.151 & .056 & .233 \\
\hline $\begin{array}{c}\text { Wins } \\
\text { Lounge }\end{array}$ & -.069 & -.111 & .109 & & & \\
\hline $\begin{array}{c}\text { Wins } \\
\text { Lab }\end{array}$ & & & & .319 & -.156 & -.141 \\
\hline \# Cigs/day & .001 & .339 & .098 & .002 & .073 & -.056 \\
\hline
\end{tabular}

Note: $\mathrm{N}=30$ for all calculations except age $\mathrm{N}=29$

Heart rate change $=$ heart rate last 30 s gambling - heart rate last 30 s baseline

SCL change $=$ skin conductance last 30 s gambling - skin conductance last 30 s baseline Arousal change $=$ arousal starting gambling - arousal stopping gambling 


\section{Appendix A}

SOGS, NODS, and CPGI scores for the past 12 month period

\begin{tabular}{|c|c|c|c|}
\hline Participant \# & SOGS & NODS & CPGI \\
\hline 27 & 0 & 0 & 2 \\
\hline 29 & 0 & 0 & 3 \\
\hline 5 & 1 & 0 & 2 \\
\hline 9 & 2 & 0 & 0 \\
\hline 11 & 2 & 0 & 2 \\
\hline 18 & 2 & 1 & 4 \\
\hline 22 & 2 & 0 & 1 \\
\hline 23 & 2 & 0 & 2 \\
\hline 10 & 3 & 0 & 6 \\
\hline 26 & 3 & 2 & 7 \\
\hline 30 & 3 & 1 & 6 \\
\hline 16 & 4 & 1 & 4 \\
\hline 28 & 4 & 0 & 6 \\
\hline 8 & 4 & 1 & 4 \\
\hline 1 & 5 & 1 & 6 \\
\hline 2 & 5 & 2 & 9 \\
\hline 6 & 6 & 3 & 14 \\
\hline 7 & 7 & 1 & 6 \\
\hline 19 & 7 & 4 & 5 \\
\hline 20 & 7 & 3 & 7 \\
\hline 21 & 7 & 1 & 7 \\
\hline 12 & 8 & 6 & 10 \\
\hline 13 & 8 & 4 & 9 \\
\hline 17 & 8 & 4 & 6 \\
\hline 24 & 9 & 5 & 6 \\
\hline 25 & 10 & 7 & 15 \\
\hline 3 & 11 & 6 & 15 \\
\hline 4 & 11 & 10 & 17 \\
\hline 14 & 13 & 10 & 27 \\
\hline 15 & 15 & 3 & 11 \\
\hline
\end{tabular}

Note: CPGI score is for Problem Gambling Severity Index

Group Means for Gambling Measures

\begin{tabular}{|c|c|c|c|c|}
\hline Measure & NPG & PPG & $t(28)$ & Significance \\
\hline SOGS & 2.3 & 8.5 & 7.5 & $<.001$ \\
& $(1.3)$ & $(2.9)$ & & \\
\hline NODS & .43 & 4.4 & 5.1 & $<.001$ \\
& $(.65)$ & $(2.8)$ & & \\
\hline CPGI & 3.5 & 10.6 & 4.3 & $<.001$ \\
& $(2.1)$ & $(5.8)$ & & \\
\hline
\end{tabular}




\begin{abstract}
Acknowledgements
Thanks to the goodwill of the management of several lounges and the helpfulness of a community sample of VLT players, we had the opportunity of being able to compare physiological and subjective responses of gamblers in natural situations and in the laboratory. We wish to extend our thanks to Mr. Al Browne, of the Red Carpet Lounge, Mr. Ron Miller, Bow Trail Boston Pizza, and Mr. Jerry Jumaga, Motel Village Boston Pizza. The assistance of Mr. Don Waldorf, President of the Alberta Gaming Industry Association, was invaluable.

This study was funded by the Alberta Gaming Research Institute. The demonstration video lottery terminal was provided by the Alberta Gaming and Liquor Commission. Kate Diskin received support from the Social Sciences and Humanities Research Council and the Alberta Heritage Foundation for Medical Research.
\end{abstract}

